\title{
RADIO SOURCE COUNTS AT 232 MHZ
}

\author{
ZHANG XIZHEN \\ Beijing Astronomical Observatory, CAS, Beijing 100080, China \\ e-mail: zxz@bao01.bao.ac.cn
}

\section{The samples}

About 30000 sources were cataloged by the Miyun $232 \mathrm{MHz}^{2}$ survey ${ }^{1}$ covering sky area north of declination $30^{\circ}$. ( Zhang et al., 1997). For source count purpose, subcounts for different ratio of signal to noise and galactic latitude of steep-spectrum and flatspectrum sources were carried out respectively.

A lots of 'new' sources were catalogued first by the Miyun survey. Some of the 'new' sources, whose flux density is less than $0.2 \mathrm{Jy}$ and no counterpart found from other catalogue, were not included in the samples. The spectral indices were derived between $232 \mathrm{MHz}$ and $4.85 \mathrm{GHz}$. The flux density of $4.85 \mathrm{GHz}$ were taken from $87 \mathrm{~GB}$ catalogue(Gregory, P.C. et al., 1990). For sources no $4.85 \mathrm{GHz}$ counterpart found, the spectrum-indices were calculated according to other frequency data. Spectra indices of 1800 sources in total were calculated in this way.

\section{Results}

The source counts were carried out by using different source groups, i.e. a) all sources, b) steep spectrum sources $\left(S \propto \nu^{\alpha}, \alpha<-0.5\right)$, c) flat spectrum sources $(\alpha>-0.5)$, d) galartic latitude $|b| \geq 10^{\circ}$, e) $|b|<10^{\circ}$.

The main results are listed as followings.

1) Results of counts on all sources and steep spectra sources are good agreement with that obtained from other low frequency survey.

2) Counts of flat spectrum sources have a wide flat region in the curves of $\log (\mathrm{dn} / \mathrm{dn} 0)$ $-\log (\mathrm{s})$. The turning-point is near $\mathbf{0 . 4} \mathrm{Jy}$ which is much lower than that of steep spectrum sources. This may show that the flat spectrum population is distributed much uniformly than the steep spectrum sources.

3) Number of the flat spectrum sources is much smaller than the number of steep sources. Mean spectral indices of them are obviously inverse correlation of their flux densities.

4) The shape of source group $|b|<10^{\circ}$ are different from the shape of sources $|b| \geq 10^{\circ}$. The low ratio of signal to noise of $|b|<10^{\circ}$ sky area may be responsible for the shape difference.

5) the Miyun catalog is complete down to about $0.25 \mathrm{Jy}$ according to the count results.

\section{References}

Gregory, P.C. et al. (1990) NRAO Preprint, 90/81

Zhang, X. et al. (1997) A. Ap.Suppl., 121, 59

${ }^{1}$ 'The project is supported by the National Nature Science Foundation of China 